\title{
Efficacy and safety of tolvaptan in hospitalised patients: a single-centre experience
}

\author{
Jesús Ruiz Ramos, Mónica Montero Hernández, María Dolores Edo Solsona, \\ Patricia Marrero Álvarez, Isabel Font Noguera, J L Poveda Andrés
}

Pharmacy Department, H.U.P. La Fe, Valencia, Spain

\section{Correspondence to} Jesús Ruiz Ramos, Pharmacy Department, H.U.P. La Fe, Avinguda de Fernando Abril Martorell, $n^{\circ} 106,46026$, Spain; jrzrms@gmail.com

Received 7 February 2014 Revised 11 June 2014 Accepted 30 June 2014 Published Online First 17 July 2014
CrossMark

To cite: Ruiz Ramos J, Montero Hernández M, Edo Solsona MD, et al. Eur J Hosp Pharm 2015;22: 28-31.

\section{ABSTRACT}

Objective To analyse the efficacy and safety of using tolvaptan and the suitability of its prescription with the criteria set forth in the pharmacotherapeutic guide of a tertiary hospital.

Method A retrospective, observational study on tolvaptan prescriptions issued in the hospital since its inclusion in the pharmacotherapeutic guide in October 2010, until February 2013. Data were collected from the patient's electronic medical records and the computerassisted prescription programme.

Results A total of 80 patients (52.3\% males) with an average age of 68.8 (SD=14.6) years were included. The most common diagnosis for its prescription was heart failure $(52.3 \%$ of cases). The initial mean for plasma sodium levels was 125.5 (SD=7.2) mEq/L. Tolvaptan use increased sodium levels to $135 \mathrm{mEq} / \mathrm{L}$ in $59.3 \%$ of patients. The median duration of treatment was 4 days (range 1-53 days). No significant differences were observed in other laboratory parameters tested. The average cost per patient was $€ 487.80$. Tolvaptan prescriptions were issued in accordance with the hospital's pharmacotherapeutic guide in $66.6 \%$ of the cases.

Conclusions Treatment with tolvaptan safely and effectively increases plasma sodium levels in patients with hyponatraemia originating for various reasons, despite its approval for use by the EMA solely for cases of hyponatraemia secondary to syndrome of inappropriate antidiuretic hormone secretion.

\section{INTRODUCTION}

Hyponatraemia, defined as plasma sodium levels of less than $135 \mathrm{mEq} / \mathrm{L}$, is the most common electrolyte disorder in hospitalised patients. ${ }^{1}$ This electrolyte disturbance has been consistently associated with an increase in morbidity and mortality, as well as longer hospital stays. ${ }^{2}$

The signs and symptoms of hyponatraemia are related to nervous system disorders caused by the entry of water into the neuronal cells from the intravascular space. Typical symptoms included nausea, vomiting, headache, muscle cramps, lethargy, anorexia and disorientation. The most serious complications occur when plasma sodium rapidly decreases to levels below $120 \mathrm{mEq} / \mathrm{L}$, a situation that may cause permanent nerve damage, respiratory arrest, coma or death. ${ }^{3}$

Hyponatraemia management depends on various factors such as the presence and severity of symptoms, its cause, whether it is presented in acute or chronic form and the individual's blood volume status. Hypovolaemic hyponatraemia is usually treated with isotonic sodium chloride $(0.9 \%)$ until the total volume has been corrected, hypervolaemic hyponatraemia with diuretics together with fluid restriction and euvolaemic hyponatraemia has traditionally been treated with hypertonic saline solution $(3 \%)$ together with diuretics. ${ }^{3}$

Syndrome of inappropriate antidiuretic hormone secretion (SIADH) is clinically manifested by euvolaemic hypoosmolar hyponatraemia. The most common causes for onset include tumours producing the antidiuretic hormone (ADH), ectopic pulmonary secretion secondary to pulmonary disease, central nervous system (CNS) disorders, general surgery or the use of certain drugs. ${ }^{4}$

Tolvaptan is the first drug authorised by the European Medicines Agency (EMA) in 2009 to treat adult patients with SIADH. Its effect is based on blocking the action of vasopressin in the V2 receptors in the distal portions of the nephron, causing an increase in aquaresis without increasing the urinary secretion of sodium. ${ }^{3} 5$ Although pivotal clinical studies included patients with hyponatraemia secondary to heart failure and cirrhosis, ${ }^{6}$ tolvaptan does not currently include these indications in its summary of product characteristics.

In 2011, various scientific societies (Spanish Society of Nephrology, Spanish Society of Endocrinology and Nutrition, Spanish Society of Internal Medicine and Spanish Society of Hospital Pharmacy) established an algorithm for the treatment of hyponatraemia, focusing on the management of euvolaemic hyponatraemia associated with SIADH. ${ }^{7}$ In accordance with this algorithm, tolvaptan is indicated for cases of hyponatraemia with sodium levels above $120 \mathrm{mmol} / \mathrm{L}$ with moderate/ mild symptoms and/or hyponatraemia lasting over $48 \mathrm{~h}$, where fluid restriction is not feasible or has failed. The recommended starting dose is $15 \mathrm{mg} /$ day, with an assessment to increase the dose to $30 \mathrm{mg} /$ day $12-24 \mathrm{~h}$ after the starting dose if the sodium increase is less than or equal to $0.4 \mathrm{mmol} /$ $\mathrm{L} / \mathrm{h}$ and plasma sodium is less than $128 \mathrm{mmol} / \mathrm{L}$ or if the total sodium increase is less than $8 \mathrm{mmol} / \mathrm{L}$ since starting the treatment. In patients where the sodium increase is less than $5 \mathrm{mmol} / \mathrm{L}$ with the $30 \mathrm{mg} /$ day dose of tolvaptan, increasing the dose to $60 \mathrm{mg} /$ day should be considered.

In our hospital, tolvaptan was included in the pharmacotherapeutic guide (PTG) in 2010 for the treatment of hyponatraemia secondary to heart failure and renal impairment without SIADH criteria, the latter two on a compassionate use basis.

The objective of this study is to analyse the use, efficacy and safety of tolvaptan and the suitability 
of its prescription against the criteria set forth in the hospital's PTG.

\section{METHODS}

Retrospective observational study on patients treated with tolvaptan in HUP $\mathrm{La} \mathrm{Fe}$ ( $\mathrm{La}$ Fe University and Polytechnic Hospital) since the inclusion of the drug in October 2010, until 2013. Demographic, diagnostic and clinical data were collected from electronic medical records and the hospital's computerassisted prescription programme.

Plasma sodium levels at the start and end of treatment were analysed in order to evaluate the efficacy of tolvaptan. Sodium levels 1 week after the end of treatment were also recorded. The efficacy of tolvaptan was compared between the different diagnoses, as well as the initial severity of hyponatraemia (severe $<130 \mathrm{mEq} / \mathrm{L}$; mild $130-135 \mathrm{mEq} / \mathrm{L}$ ). The influence of the concomitant use of tolvaptan with furosemide and fluid restriction in the increase of plasma sodium levels was also studied.

The safety of tolvaptan was evaluated by analysing the possible onset of laboratory disorders classified as common in the summary of product characteristics: increase in alanine transaminase (ALT or GPT) and aspartate transaminase (AST or GOT) liver enzymes, hyperglycaemia, hyperkalaemia, hypernatraemia, hyperuricaemia, hypercalcaemia and increase in plasma creatinine levels.

Furthermore, we assessed the degree of suitability of the prescriptions in the treatment algorithm for hyponatraemia, as established by the Spanish Society of Nephrology, Endocrinology and Nutrition, Internal Medicine and Hospital Pharmacy ${ }^{7}$ and the criteria established by the Pharmacological and Therapeutic Committee for its inclusion in the hospital's PTG. In order to do so, we analysed the diagnosis for which tolvaptan was prescribed, as well as the laboratory measurements requested to confirm the diagnosis of SIADH.

The laboratory results were analysed using the software SPSS V.15.0. Qualitative variables were expressed as percentages, while the mean and the SD or median, and percentiles $25-75$, were used to describe the variables. Student's $t$ test was used in order to compare the increase in sodium levels associated with the use of tolvaptan, as well as the changes in the laboratory variables and the efficacy based on the initial severity of hyponatraemia. Analysis of variance was used to compare the efficacy between diagnoses.

\section{RESULTS}

During the study period, 80 patients received treatment with tolvaptan, of which 6 received treatment during different hospital admissions. The demographic and clinical characteristics of the patients studied are shown in table 1 . The maximum dose prescribed was $15 \mathrm{mg} /$ day in $89.5 \%$ of patients, $30 \mathrm{mg} /$ day in $8.1 \%$ and $7.5 \mathrm{mg} /$ day in $2.3 \%$. The median treatment duration was 4 days (range 1-45 days). Twelve patients received one single dose of tolvaptan, while three patients remained on the treatment for more than 30 days. Two patients continued treatment with tolvaptan after hospital discharge.

The mean value of plasma sodium prior to starting treatment was $125.5(\mathrm{SD}=7.2) \mathrm{mEq} / \mathrm{L}$, with $64.0 \%$ of the patients presenting severe hyponatraemia $(\mathrm{Na}<130 \mathrm{mEq} / \mathrm{L})$. Eight patients presented plasma $\mathrm{Na}$ values of greater than $135 \mathrm{mEq} / \mathrm{L}$ on the day when treatment with tolvaptan was started.

With regards to the tests performed during treatment, only plasma sodium measurements were carried out in $47.7 \%$ of the patients, while another additional test was carried out in the
Table 1 Demographic and clinical characteristics of patients of the study $(\mathrm{n}=80)$

\begin{tabular}{lc}
\hline Mean age (SD) & $70.5(11.5)$ \\
Sex (\%) & 52.3 \\
Male & 47.7 \\
Female & \\
Main diagnosis (\%) & 52.3 \\
Chronic heart failure & 12.8 \\
SIADH & 14.0 \\
Cirrhosis & 7.0 \\
Others & \\
Prescriber service (\%) & 40.7 \\
Cardiology & 20.0 \\
Hepatology & 10.5 \\
Internal medicine & 7.0 \\
Nephrology & 21.8 \\
Others & \\
\hline SIADH, syndrome of inappropriate antidiuretic hormone secretion. &
\end{tabular}

remaining 52.3\%: urine sodium in $17.4 \%$ of the patients, plasma osmolarity in $20.9 \%$ and urine osmolarity in $13.9 \%$.

Tolvaptan was associated with a mean increase in sodium levels of $9.5(\mathrm{SD}=7.8) \mathrm{mEq} / \mathrm{L}$ (figure 1), making it able to normalise plasma sodium levels (values between 135 and $145 \mathrm{mEq} / \mathrm{L}$ ) in $53.3 \%$ of the patients and to maintain sodium levels around $130 \mathrm{mEq} / \mathrm{L}$ in $74.4 \%$ of the patients studied. The increase in sodium levels was $11.4(\mathrm{SD}=7.3 \mathrm{mEq} / \mathrm{L})$ in those patients with severe hyponatraemia and $4.5(5.0) \mathrm{mEq} / \mathrm{L}$ in patients with mild hyponatraemia. Eight patients with mild hyponatraemia did not present an increase in sodium levels after receiving treatment with tolvaptan. One week after treatment, sodium levels decrease in 24 patients, reaching levels below $130 \mathrm{mEq} / \mathrm{L}$ in eight patients.

There was no significant difference $(\mathrm{p}=0.376)$ in the increase of sodium levels between patients with SIADH, heart failure and cirrhosis. Furosemide together with tolvaptan was prescribed in $69.7 \%$ of the patients, without any significant differences being found in the increase of plasma sodium versus the group of patients who did not receive furosemide $(p=0.979)$. Furthermore, no significant differences were found in the group of patients with fluid restriction $(26.7 \%)$ and without it $(\mathrm{p}=0.761)$.

Treatment with tolvaptan showed no significant effect on the glucose, uric acid, potassium, calcium or creatinine values in the patients treated (table 2).

No significant increases in the liver enzymes were observed with treatment with tolvaptan. In the three patients with a treatment duration of over 30 days, no increase was observed in the AST/ALT liver enzyme values between the start and end of treatment (mean: $\mathrm{AST}=42.6$ vs $27.0 \mathrm{IU} / \mathrm{L} ; \mathrm{ALT}=27.0$ vs $25.3 \mathrm{IU} / \mathrm{L}$ ). In patients with cirrhosis, the use of tolvaptan was also not associated with a significant increase in liver enzymes (AST $=55.3$ vs $54.6 \mathrm{IU} / \mathrm{L} ; \mathrm{ALT}=29.6$ vs $30.6 \mathrm{IU} / \mathrm{L}$ ).

Regarding the suitability of the prescriptions for the treatment algorithm, $88.8 \%$ of the patients with hyponatraemia complied with the requirements set forth in the prescription in terms of the starting dose of $15 \mathrm{mg}$ and a dose increase based on sodium plasma levels. Three patients received a maximum dose of tolvaptan of $7.5 \mathrm{mg}$, a dose not taken from the algorithm, and only one patient started treatment with a dose of $30 \mathrm{mg}$. Twenty per cent of the patients started tolvaptan with initial sodium levels of less than $120 \mathrm{mmol} / \mathrm{L}$. Additionally, $26 \%$ of the patients started treatment with tolvaptan with instructions to 


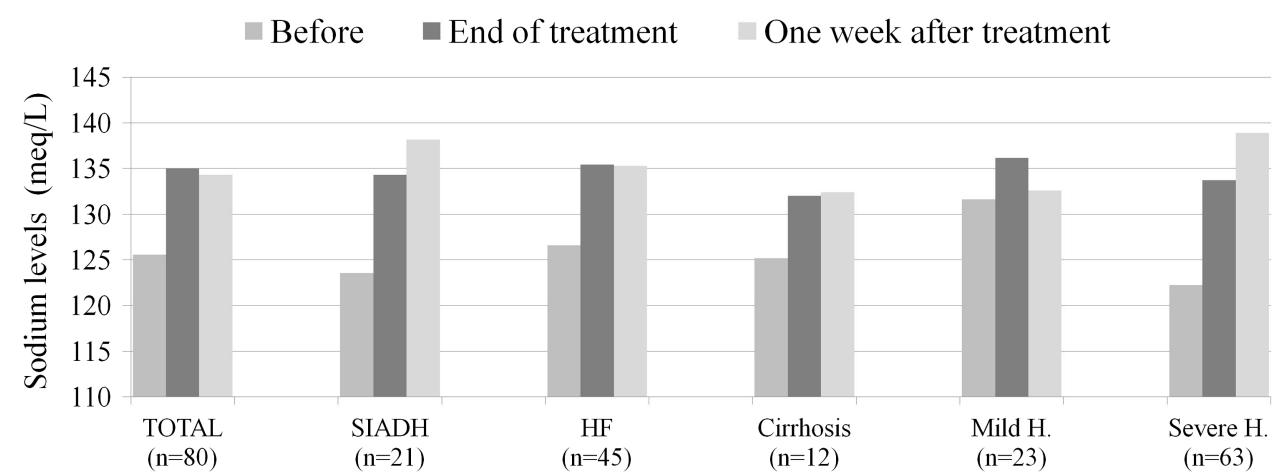

Figure 1 Variation in sodium levels after tolvaptan treatment ( $p<0.05$ at the end of treatment for all comparisons). HF, heart failure; $\mathrm{H}$, hyponatraemia.

maintain fluid restriction, a measure not recommended in the algorithm.

Of the eight patients who did not present an increase in sodium levels with treatment with tolvaptan, none received the maximum dose of $60 \mathrm{mg} /$ day.

The prescription of tolvaptan was in compliance with the provisions set forth in the hospital's PTG in $63.3 \%$ of the cases, mostly being used for heart failure. Tolvaptan was prescribed in six patients despite them having a clinical condition in which its administration was not recommended (anuria), while in eight patients tolvaptan was prescribed with sodium levels greater than $135 \mathrm{mEq} / \mathrm{L}$. Of these, three patients did not present an increase in sodium levels during the treatment.

No significant difference was observed in the increase of sodium levels between the prescriptions in accordance with the PTG and those for which a prescription was provided outside of these criteria (9.54 vs $9.41 \mathrm{mEq} / \mathrm{L} ; \mathrm{p}=0.684)$.

The average cost of treatment with tolvaptan was $€ 487.76$ (95\% CI $€ 355.90$ to 619.50) per hospitalised patient.

\section{DISCUSSION}

The study data are consistent with those reported in previous studies. ${ }^{89} 10$ Tolvaptan is an effective drug for increasing plasma sodium levels in hospitalised patients and shows a good safety profile. Despite this, the economic cost per patient is high.

The limitations of our study include the lack of comparison with other measures used in patients with hyponatraemia and that the most common adverse effects, such as dry mouth and thirst, could not be confirmed.

The introduction of vasopressin antagonists is an attractive option for managing hyponatraemia. Initial studies showed good

Table 2 Laboratory results studied before and after tolvaptan treatment

\begin{tabular}{lccc}
\hline & $\begin{array}{c}\text { Mean (SD) levels } \\
\text { before treatment }\end{array}$ & $\begin{array}{c}\text { Mean (SD) levels } \\
\text { after treatment }\end{array}$ & p Value \\
\hline Potassium (mEq/L) & $4.4(0.8)$ & $4.3(0.7)$ & 0.136 \\
Glucose (mg/dL) & $114.3(48.1)$ & $116.2(54.8)$ & 0.871 \\
Creatinine (mg/dL) & $1.4(0.9)$ & $1.4(1.1)$ & 0.733 \\
Calcium (mg/dL) & $8.5(0.8)$ & $8.8(0.8)$ & 0.190 \\
Uric acid $(\mathrm{mg} / \mathrm{dL})$ & $6.9(3.1)$ & $7.2(2.9)$ & 0.359 \\
AST/GOT $(\mathrm{UI} / \mathrm{mL})$ & $41.9(35.1)$ & $36.2(23.1)$ & 0.070 \\
ALT/GPT $(\mathrm{UI} / \mathrm{mL})$ & $33.2(56.5)$ & $31.0(40.5)$ & 0.456 \\
\hline
\end{tabular}

efficacy and safety in the treatment of moderate hyponatraemia. In the SALT- 1 and SALT-2 pivotal studies, ${ }^{5}{ }^{6}$ it was observed that plasma sodium was effectively restored in patients with hyponatraemia associated with SIADH, cirrhosis or heart failure, as well as with mild or severe hyponatraemia. In the EVEREST trial, which assessed the efficacy of tolvaptan in 4133 patients with heart failure, an adequate safety profile was also obtained. ${ }^{11}$ Other case series studies have shown similar results in this group of patients, showing an adequate safety profile. ${ }^{10} 12$

Due to the results obtained in the trials on patients with autosomal-dominant polycystic kidney disease, in which an increase in the risk of hepatotoxicity was observed, the US Food and Drug Administration has recently established a recommendation to not exceed 30 days of treatment and avoid use in patients with cirrhosis. ${ }^{13}$ Nevertheless, the doses used in this group of patients exceed $60 \mathrm{mg} / \mathrm{day},{ }^{14}$ doses that are much higher than those used in the treatment of hyponatraemia with SIADH. No increase in liver enzymes was observed in our study, either in the patients who exceeded a duration of 30 days (three patients) or in the group of patients with cirrhosis (12 patients).

The low prevalence of requests for plasma and urine osmolarity tests is to be noted. These tests were only requested in $10.5 \%$ of the patients, which were necessary to confirm the diagnosis of SIADH.

Hyponatraemia associated with heart failure or cirrhosis, despite being an inclusion criterion in the pivotal trials for the authorisation of the drug, ${ }^{15}$ is not an indication authorised for tolvaptan use. Despite this, hyponatraemia associated with chronic heart failure is a common reason for prescribing tolvaptan in our hospital.

The increase in sodium levels in the study patients with hyponatraemia associated with heart failure has been significant, with no differences in the efficacy and safety of the drug being found in relation to patients with hyponatraemia secondary to SIADH. Despite this, the potential clinical benefits of tolvaptan in patients with heart failure associated with hyponatraemia have not yet been demonstrated, ${ }^{16}$ for which reason its use should be monitored in this group of patients.

On the other hand, published studies on cirrhosis have not shown particularly beneficial effects. A recent meta-analysis shows that, despite mortality not being affected, the use of vaptans in these patients has not been able to reduce mortality, nor variables such as bleeding varices, hepatic encephalopathy or renal failure. ${ }^{17}$ In the subgroup of patients with cirrhosis in our study, an increase in plasma sodium levels was indeed observed. However, clinical variables related to this effect were not measured. 
Other vasopressin receptor antagonists have been studied for the treatment of hypervolaemic and euvolaemic hyponatraemia. Conivaptan, a non-selective V1/V2 vasopressin receptor antagonist, administered intravenously, is now marketed in the USA, while other drugs such as mozavaptan, lixivaptan and satavaptan, administered by oral route, are under clinical development.

The efficacy of the vaptans seems to be well established for the short-term management of hyponatraemia. However, its efficacy against long-term morbidity and mortality in patients with heart failure and cirrhosis has not yet been well defined. ${ }^{18}$ Further studies are necessary in these groups of patients.

The cost of tolvaptan per patient has been high in comparison with the traditional measures established for managing hyponatraemia, such as fluid restriction or the administration of hypertonic saline solution. However, hyponatraemia has been associated with an increase in the mean time of hospitalisation, ${ }^{19}$ for which reason its rapid correction could lead to a reduced hospital stay, and therefore, a reduced cost. ${ }^{20}$ Based on the reduced hospital stay associated with tolvaptan, as reported in the SALT-1 and SALT-2 clinical trials, one study associated the use of tolvaptan with a cost reduction of $\$ 694$ per patient treated. ${ }^{21}$ However, further studies are needed that show correction between the rapid recovery from hyponatraemia with a reduced hospitalisation time compared with the traditional options available such as hypertonic saline or fluid restriction in order to assess the true economic impact of this drug.

The increased incidence of hyponatraemia in hospitalised patients and the lack of experience in non-SIADH indications mean that it is necessary to establish strict prescription criteria or protocols for use to guarantee the rational use of tolvaptan in hospitals.

\section{What this paper adds}

- Hyponatraemia affects $15-20 \%$ of hospitalised patients, and, in addition to being a severity marker, it contributes to worsening the underlying disease. In the SALT-1 and SALT-2 pivotal studies and in the EVEREST trial, it was observed that plasma sodium was effectively restored with tolvaptan in patients with hyponatraemia associated with syndrome of inappropriate antidiuretic hormone secretion (SIADH), cirrhosis or heart failure.

- Given the possibility of a new form of management for patients with hyponatraemia after the inclusion of tolvaptan into common medical care since its approval by the European Medicines Agency, we have conducted a retrospective, observational study of its efficacy, safety and the suitability of its prescription in the various hospital units.

- Our report conclude that treatment with tolvaptan safely and effectively increases plasma sodium levels in patients with hyponatraemia originating for various reasons, despite its approval for use by the EMA solely for cases of hyponatraemia secondary to SIADH.
Competing interests None.

Ethics approval

Provenance and peer review Not commissioned; externally peer reviewed.

\section{REFERENCES}

1 Upadhyay A, Jaber BL, Madias NE. Incidence and prevalence of hyponatremia. Am J Med 2006;119(7 Suppl 1):S30-35.

2 Gill G, Huda B, Boyd A, et al. Characteristics and mortality of severe hyponatraemia —a hospital-based study. Clin Endocrinol (Oxf) 2006;65:246-9.

3 Deitelzweig SB, McCormick L. Hyponatremia in hospitalized patients: the potential role of tolvaptan. Hosp Pract (1995) 2011;39:87-98.

4 Sherlock M, Thompson CJ. The syndrome of inappropriate antidiuretic hormone: current and future management options. Eur J Endocrinol 2010;162(Suppl 1): S13-18.

5 Schrier RW, Gross P, Gheorghiade M, et al. Tolvaptan, a Selective Oral Vasopressin V2-Receptor Antagonist, for Hyponatremia. N Engl J Med 2006;355:2099-112.

6 Runkle I, Villabona C, Navarro A, et al. Algoritmo de tratamiento de la hiponatremia. http://www.senefro.org/modules/webstructure/files/algoritmo_ hiponatremia_2012.pdf

7 Shoaf SE, Bramer SL, Bricmont P, et al. Pharmacokinetic and pharmacodynamic interaction between tolvaptan, a non-peptide AVP antagonist, and furosemide or hydrochlorothiazide. J Cardiovasc Pharmacol 2007;50:213-22.

8 Rajendran R, Grossman AB, Kar P. Vasopressin receptor antagonist in the treatment of the syndrome of inappropriate antidiuretic hormone in general hospital practice. Endocr J 2012;59:903-9.

9 Gheorghiade M, Gottlieb SS, Udelson JE, et al. Vasopressin v(2) receptor blockade with tolvaptan versus fluid restriction in the treatment of hyponatremia. $\mathrm{Am} \mathrm{J}$ Cardiol 2006:97:1064-7.

10 Rodríguez-de Muñoz YM, Sánchez-Lázaro IJ, Almenar-Bonet L, et al. Use of Tolvaptan in Patients With Hyponatremia Due to Heart Failure: Initial Experience. Rev Esp Cardiol 2013;66:319-21.

11 Gheorghiade M, Konstam MA, Burnett JC, et al. Short-term clinical effects of tolvaptan, an oral vasopressin antagonist, in patients hospitalized for heart failure: the EVEREST Clinical Status Trials. JAMA 2007;297:1332-43.

12 Salterain-Gonzalez N, Esteban-Fernández A, García-López M, et al. Efficacy of tolvaptan in patients hospitalized for heart failure with refractory hyponatremia. Clinical experience in daily practice. Rev Esp Cardiol Engl Ed 2013;66:503-4

13 Samsca (Tolvaptan): Drug Safety Communication-FDA Limits Duration and Usage Due To Possible Liver Injury Leading to Organ Transplant or Death. US Food and Drug Administration. Disponible en http://www.fda.gov/Safety

14 Higashihara E, Torres VE, Chapman AB, et al. Tolvaptan in Autosomal Dominant Polycystic Kidney Disease: Three Years' Experience. Clin J Am Soc Nephrol 2011:6:2499-507.

15 Gross PA, Wagner A, Decaux G. Vaptans are not the mainstay of treatment in hyponatremia: perhaps not yet. Kidney Int 2011;80:594-600.

16 Zmily HD, Daifallah S, Ghali JK. Tolvaptan, hyponatremia, and heart failure. Int J Nephrol Renovasc Dis 2011;4:57-71.

17 Dahl E, Gluud LL, Kimer N, et al. Meta-analysis: the safety and efficacy of vaptans (tolvaptan, satavaptan and lixivaptan) in cirrhosis with ascites or hyponatraemia. Aliment Pharmacol Ther 2012;36:619-26.

18 Narayen G, Mandal SN. Vasopressin receptor antagonists and their role in clinical medicine. Indian J Endocrinol Metab 2012;16:183-91.

19 Deitelzweig S, Amin A, Christian R, et al. Hyponatremia-Associated Healthcare Burden Among US Patients Hospitalized for Cirrhosis. Adv Ther 2013;30:71-80

20 Grant P. New drugs for hyponatraemia. Cost effectiveness of tolvaptan. BMJ 2011;342:d1947.

21 Dasta JF, Chiong JR, Christian R, et al. Evaluation of costs associated with tolvaptan-mediated hospital length of stay reduction among US patients with the syndrome of inappropriate antidiuretic hormone secretion, based on SALT-1 and SALT-2 trials. Hosp Pract (1995) 2012;40:7-14. 